\begin{tabular}{lll}
\hline AECORDS OF PHARMACEUTICAL \\
AND BIOMEDICAL SCIENCES
\end{tabular}

\title{
Biochemical linkage of type 2 diabetes mellitus and Alzheimer's disease
}

\author{
Samar S. Khalaf ${ }^{a *}$, Mohamed M. Hafez ${ }^{b}$, Eman T. Mehanna ${ }^{a}$, Noha M. Mesbaha, Dina M. Abo-Elmatty \\ ${ }^{a}$ Department of Biochemistry, Faculty of Pharmacy, Suez Canal University, Ismailia, Egypt \\ ${ }^{b}$ Department of Biochemistry, Faculty of Pharmacy, Ahram Canadian University, October 6 City, Egypt
}

Received on: 18.04. 2019

Revised on: 29. 04. 2019

Accepted on: 05. 05. 2019

Correspondence Author:

Tel:+ 201025888320

E-mail address:

samarsamir483@yahoo.com

\begin{abstract}
Type 2 diabetes mellitus (T2DM) is a common disease in the elderly, affecting around $20 \%$ of geriatrics. In cross-sectional studies, T2DM had various adverse health effects, including cognitive impairment. The association of T2DM with decreased cognitive function suggests that T2DM can contribute to Alzheimer's disease (AD)

The relationship between $\mathrm{T} 2 \mathrm{DM}$ and $\mathrm{AD}$ continues to grow rapidly. It has been suggested that AD can be considered "type 3 diabetes". Along with the processing of amyloid precursor protein (APP) and tau phosphorylation, the molecular links between T2DM and $\mathrm{AD}$ provide clues for new therapeutic targets such as glucagon-like peptide-1 (GLP-1), butyrylcholinesterase and receptor for advanced glycosylation end products (AGE).

A possible mechanism correlating $\mathrm{T} 2 \mathrm{DM}$ and $\mathrm{AD}$ is the alteration in insulin signaling in the brain. Insulin signaling is involved in several neuronal functions, and plays a vital role in the pathophysiology of AD. Therefore, the modification of neuronal insulin signaling by diabetic conditions may contribute to $\mathrm{AD}$ progression. Another possible mechanism is cerebrovascular changes, a common pathological change observed in both diseases. The importance of amyloid beta peptide (A $\beta)$ induced cerebrovascular dysfunction in $\mathrm{AD}$ has been reported, indicating that pathological interactions between AGE receptor and $A \beta$ have a role in this disorder.
\end{abstract}

Keywords: Alzheimer's disease; diabetes mellitus; amyloid beta peptide; tau protein.

\section{Introduction}

Diabets mellitus (DM) and Alzheimer disease (AD) are both common and increasing in incidence in the aging population. $\mathrm{AD}$ is characterized by progressive cognitive decline and accumulation of $\beta$-amyloid (A $\beta$ ) forming senile plaques. $\mathrm{DM}$ is a metabolic disorder characterized by hyperglycemia in the context of insulin resistance and relative lack of insulin. Both diseases also share common characteristics such as loss of cognitive function and inflammation. Inflammation resulting from $A \beta$ further induces production of $A \beta_{1-42}$ peptides. Inflammation due to overnutrition induces insulin resistance and consequently DM (de Nazareth, 2017).

Recent evidence has shown common pathogenic factors operating in both conditions. Diabetes causes neurodegeneration by inducing changes in vascular function and structure, glucose metabolism, and insulin signaling. In turn, $\mathrm{AD}$ influences systemic glucose metabolism by inducing behavioral changes, memory disturbances 
and hypothalamic dysfunction. Hypoglycemia, one of the major conditions encountered during the treatment of patients with diabetes, may also contribute to neurodegeneration. Through this vicious circle, diabetes and $\mathrm{AD}$ may cooperate to cause neurodegeneration. Various molecular, cellular, inter-organ, physical and clinical factors might contribute to the bidirectional interactions between diabetes and AD (Mitsuru and NaoyukiSato, 2017).

\section{Type 2 Diabetes mellitus}

T2DM is a progressive disease defined by insulin resistance that eventually leads to deficiency of insulin secretion. Although the etiology of T1DM and T2DM varies significantly, both lead to hyperglycemia and share macrovascular (coronary, cerebrovascular and peripheral vascular) and microvascular (retinopathic, nephropathic and neuropathic) complications. T2DM is usually diagnosed by screening. A diagnosis of T2DM is made when fasting plasma glucose is greater than $126 \mathrm{mg} / \mathrm{dL}(7 \mathrm{mmol} / \mathrm{L})$ on two separate tests indicates diabetes, and $\mathrm{HbA} 1_{\mathrm{C}}$ levels are greater than of 6.5 percent. $\mathrm{HbA} 1_{\mathrm{C}}$ between 5.7 and 6.4 percent indicates prediabetes, while a value of HbA1c less than 5.7 is normal (Rodriguez et al., 2005, American Diabetes Association, 2012).

The genetic predisposition to T2TDM is often high. Insulin resistance is associated with sedentary lifestyle and overweight (BMI $25-29.9 \mathrm{~kg} / \mathrm{m}^{2}$ ) or obesity (BMI > $\left.30 \mathrm{~kg} / \mathrm{m}^{2}\right)$.Weight loss often reduces insulin resistance in obese patients and can delay the onset of diabetes or improve its severity and risk of long-term complications. Insulin resistance mainly affects the liver, muscle and adipocytes and is characterized by complex disturbances in cell receptors, function of glucokinase and other metabolic processes (Ye, 2013).

The main drivers of the current diabetes epidemic appear to be lifestyle (obesity, physical inactivity and stress). At least $80 \%$ of T2DM cases can be prevented by management of obesity in all age groups. Diabetes progression in prediabetic states can be reduced by 50 percent by modest weight loss using diet and regular physical activity (Knowler et al., 2009).

In addition, several pharmacological agents, including metformin, thiazolidinedione and alpha glycosidases inhibitors, reduce the progression of prediabetes to diabetes. Change in lifestyle is the preferred method for most patients (McMurray et al., 2010).

All types of diabetes increase the risk of long-term complications. These usually develop after many years (10 - 20), but may be the first symptom in people who have not been diagnosed. The main long-term complications are related to blood vessel damage. Diabetes doubles the risk of heart disease. Ischemic heart disease (angina pectoris and myocardial infarction), stroke and peripheral vascular disease are the major macrovascular (related to atherosclerosis of large arteries) diseases. The capillaries also suffer from microangiopathy secondary to diabetes. Diabetic retinopathy, which affects the formation of blood vessels in the retina of the eye, may cause visual symptoms, including reduced vision and potentially blindness. Diabetic nephropathy, the effect of diabetes on the kidneys, can result in scarring changes in the renal tissue, proteinuria, and possibly chronic renal disease requiring dialysis (Michael and Fowler, 2008; Chawla et al., 2016).

Another complication is diabetic neuropathy, causing numbness, tingling and pain in the feet, and increasing the risk of skin damage. Along with vascular disease in the legs, neuropathy exacerbates diabetes-related foot problems (such as diabetic foot ulcers) that may be difficult to treat and may require amputation. Proximal diabetic neuropathy also causes painful muscle waste and weakness. There is a link between diabetes and cognitive impairment. Compared to those without diabetes, diabetics have a 1.2 to 1.5 fold greater rate of decline in cognitive function (Paisley and Serpell, 2017). Diabetes mellitus is considered a risk factor for vascular dementia. This type of dementia is caused by brain damage that is often caused by reduced or blocked blood flow to the brain. Many people with diabetes have brain changes that are characteristic of both $\mathrm{AD}$ and vascular dementia (Luchsinger, 2010).

\section{Alzheimer's disease}

\subsection{Symptoms of Alzheimer's disease}

Alzheimer' disease (AD) is a chronic neurodegenerative disease with an insidious onset and gradual but slow decline. It is the most common form of dementia. According to estimates from the United Nations, the number of people aged 60 or above is 962 million in 2017 and over 
two-thirds live in low- and middle-income countries. This is expected to increase to 2.1 billion by 2050 , with nearly $80 \%$ living in low- and middle-income countries (Prina et al., 2019).

It often coexists with vascular dementia, the most common type of mixed dementia. The AD histopathology is characterized by senile plaques, neurofibrillary tangles and neuronal loss (Grossman et al., 2006; Serrano-Pozo et al., 2011).

The first symptom is short term memory loss. As the disease progresses, symptoms may include problems with language, disorientation, mood swings, loss of motivation, not managing self-care, and behavior problems. As a person's condition declines, he often withdraws from family and society. Bodily functions are lost gradually, ultimately leading to death. Although the rate of progression can vary, the average life expectancy after diagnosis is three to nine years (Burns and Iliffe, 2009).

\subsection{Causes of Alzheimer's disease}

Although gross histological features of AD in the brain are well characterized, three hypotheses have been proposed regarding the main cause. The oldest hypothesis suggests that deficiency in cholinergic signaling initiates the progression of the disease. Two alternative hypotheses suggest that misfolding of tau or $\mathrm{A} \beta$ proteins initiates the cascade (Du et al., 2018).

There are several theories behind the formation of senile plaques and neurofibrillary tangles. The amyloid hypothesis is currently the most commonly accepted explanation. The brains of Alzheimer's patients have an excess of amyloid interneuron peptides, due to overproduction or decreased clearance of $A ß$. This leads to the formation of dense amyloid oligomers, which are deposited in the form of diffuse plaques. These plaques cause an inflammatory process through activation of the microglia, formation of cytokines and activation of the complement cascade. Inflammation results in the formation of senile plaques, causing synaptic and senile injury and cell death (Reitz 2012) (Fig. 1).

A second theory is that $\mathrm{AD}$ is caused by abnormal aggregation of tau protein. Tau is a protein that regulates axonal microtubule stability.
According to the current $\mathrm{AD}$ assumptions, the tau protein is abnormally phosphorylated, dissociates from microtubules, and aggregates in neurofibrillary tangles (NFT). Tau has at least 45 phosphorylation sites, most of them are located in the proline rich region (P-region) (172-251 residues) and the region of the $\mathrm{C}$-terminal tail (region C) (residues 368 -441). Tau phosphorylation in these two regions affects its ability to interact with microtubules (Hanger et al., 2009). In terms of developing $\mathrm{AD}$, phosphorylation sites in the C-terminal region seems to play a major role. Phosphorylation at Ser262 selectively alters the binding of tau to microtubules. Phosphorylation at Ser202 improves the polymerization of tau, and phosphorylation at Ser202-Thr205 makes the formation of filaments more sensitive to small changes in the concentration of tau. Given these data together, it appears that several phosphorylation events of tau, rather than the singular phosphorylation, play a crucial role in the AD-related tau pathology (Rankin et al., 2005; Mondragón-Rodríguez et al., 2013).

The "Cholinergic hypothesis" states that AD begins with deficiency of acetylcholine, a vital neurotransmitter. The first therapeutic research was based on this assumption, including the restoration of the "cholinergic nuclei. First-generation antiAlzheimer medicines works to preserve acetylcholine (Ach) by inhibiting acetylcholinesterases (AChE). While sometimes beneficial, these drugs did not lead to a cure. They were used to treat the disease's symptoms but do not stop or reverse it. All pre- and post-ganglionic parasympathetic neurons and all pre-ganglionic sympathetic neurons use ACh as a neurotransmitter. Given its widespread distribution in the brain, it is not surprising that cholinergic neurotransmission is responsible for modulating important neural functions. The cholinergic system is involved in critical physiological processes, such as attention, learning, memory, stress response, wakefulness, sleep, and sensory information. Deficits in the cholinergic transmission can potentially influence all aspects of cognition and behavior, including cortical and hippocampal processing information. Cholinergic effects were proposed as a potential causative agent of plaque and tangle formation, leading to widespread neuroinflammation (Ferreira-Vieira et al., 2016). 


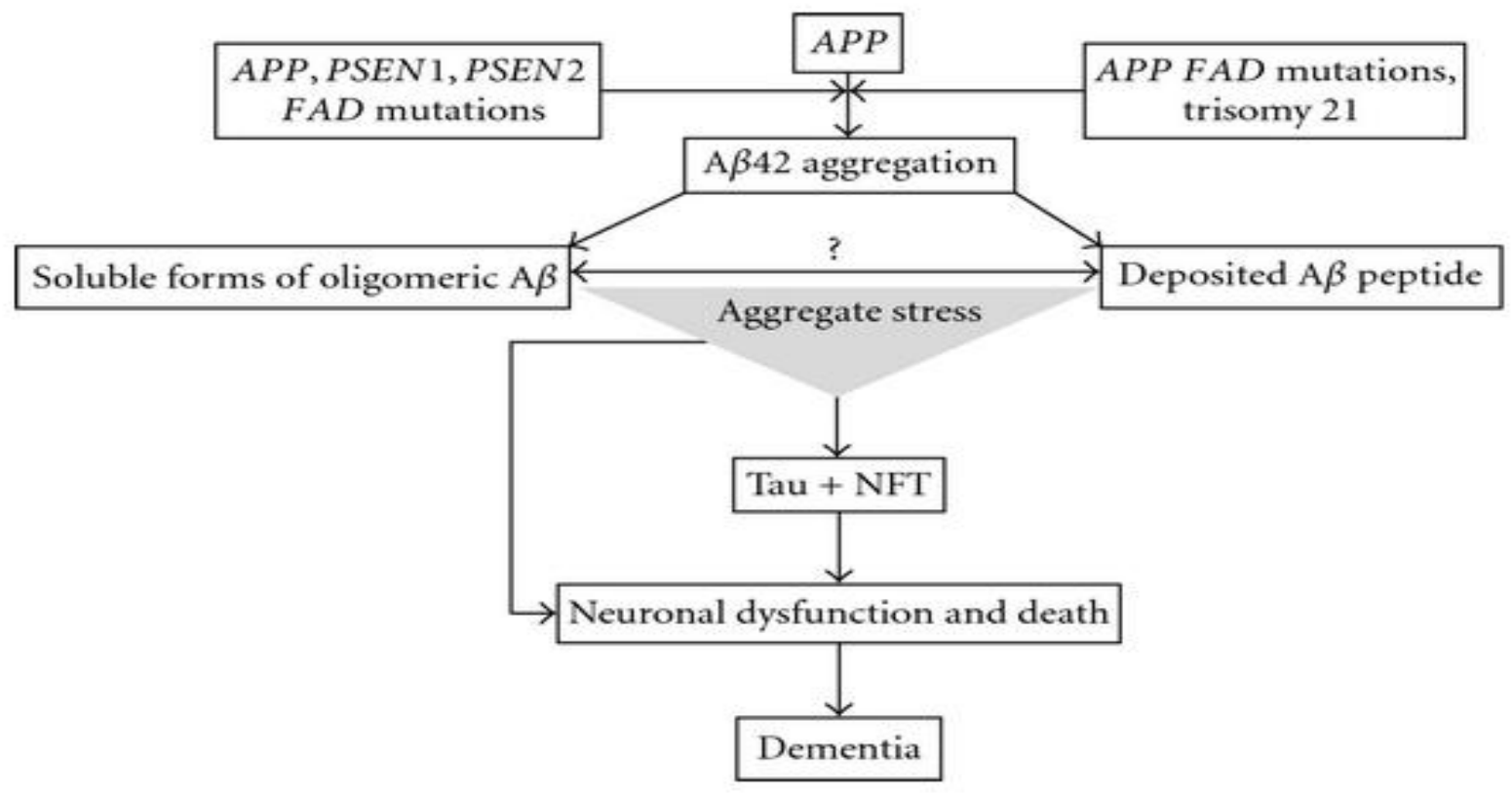

Figure 1. The amyloid cascade hypothesis (Reitz 2012). APP; amyloid precursor protein, PSEN; presnilin, FAD; familial Alzheimer disease, Aß; amyloide beta peptide, NFT; neurofilbrillary tangles.

\subsection{Risk factors of Alzheimer's disease}

Identification of risk factors for $\mathrm{AD}$ is essential to develop effective diagnostic and therapeutic strategies. They include:

\section{a) Advanced age}

The greatest known risk factor for $\mathrm{AD}$ is increasing age (Xue et al., 2017). About 3\% of people between the ages of 65-74 have dementia, $19 \%$ between 75 and 84 , and nearly half of those over 85 years of age. In 2013 dementia resulted in about 1.7 million deaths, up from 0.8 million in 1990 (GBD 2013 Mortality and Causes of Death Collaborators, 2015). AD is subdivided into early onset (EOAD) and late onset (LOAD). EOAD has an onset before age $60-65$ years and accounts for $1-5 \%$ of all cases. LOAD has an onset after age 60-65 years and is the predominant form of AD (Goldman et al., 2011).

\section{b) Family history and genetics}

Although most cases of AD are sporadic (not inherited), familial forms of AD exist. Subjects with a family history of AD had lower baseline scores in processing speed, executive functioning, memory encoding, and delayed memory when compared with those without a family history (Donix et al., 2012). Additionally, family history may be consistent with autosomal dominant, familial, or sporadic AD. Families with autosomal dominant $\mathrm{AD}$ are characterized by disease that occurs in at least three individuals in two or more generations, with two of the individuals being first-degree relatives of the third (e.g., grandparent, parent, and child). Familial AD is characterized by disease that occurs in more than one individual, and at least two of the affected individuals are third-degree relatives or closer. Familial clustering represents approximately $15-25 \%$ of AD cases. Sporadic AD is characterized by an isolated case in the family or cases separated by more than three degrees of relationship. Sporadic AD represents approximately $75 \%$ of all cases. Currently, there are three identified causative genes in which mutations are associated with autosomal dominant EOAD: presnilin (PSEN1, PSEN2), and APP (Goldman et al., 2011).

\section{c) Down syndrome}

People with Down syndrome (DS) are at high risk of developing $\mathrm{AD}$ at a relatively young age. (Castro et al., 2016) Given the location of the APP gene on chromosome 21 , the hypothesis of the amyloid cascade is the prevailing theory accounting for this risk, with other genetic and environmental factors affecting age of onset and course of the disease (Head et al., 2016).

\section{d) Cerebrovascular disease}

Although $\mathrm{AD}$ is classified as a neurodegeneration 
cerebrovascular disease, there is evidence that vascular risk factors and CVD may accelerate $A \beta$ 40-42 production/ aggregation/deposition and contribute to the pathology and symptomatology of AD. A $\beta$ deposited along the vessels also causes cerebral amyloid angiopathy. The role of vascular factors and $\mathrm{A} \beta$ emphasize that vascular risk factor management is important for $\mathrm{AD}$ prevention and treatment (Honjo et al., 2012).

\section{e) Depression and gender}

Depression is associated with an increased risk of dementia and $\mathrm{AD}$, although it is unclear if it is a real risk factor or a prodrome. The impact of depression on development of dementia and $\mathrm{AD}$ varies by gender. Premorbid depressive symptoms were found to significantly increase the risk of dementia, especially $\mathrm{AD}$, in men but not in women (Dal Forno et al., 2005; Quattropani et al., 2018).

\section{f) Diabetes mellitus}

There is evidence of a link between T2DM and cognitive decline. T2DM has been identified as a risk factor for $\mathrm{AD}$. A common observation for both conditions is the desensitization of insulin receptors in the brain. Insulin acts as a growth factor in the brain and is neuroprotective. It activates dendritic sprouting, regeneration and proliferation of stem cells. The loss of effect of insulin can facilitate the development of AD. Several drugs have been developed to treat T2DM, which re-sensitize the insulin receptors and may be useful in preventing the neurodegenerative processes in the brain. In particular, incretins GLP-1 and glucose-dependent polypeptide insolinotropic (GIP) are hormones that re-sensitize the insulin signaling. The incretins also have growth factor similar properties as insulin and are neuroprotective. In mouse models of AD, GLP1 receptor agonists reduce amyloid plaque formation, reduce the inflammation response in the brain, protect neurons from oxidative stress, induce neurite outgrowth, and protect synaptic plasticity and memory formation from the detrimental effects caused by $A \beta$ production and inflammation. Other growth factors such as brainderived neurotrophic factor (BDNF), nerve growth factor (NGF), or growth factor insulin-like 1 (IGF1) also showed a range of neuroprotective properties in preclinical studies.These growth factors activate cell signaling mechanisms that are protective and regenerative, which suggests that the initial process that may trigger the cascade of neurodegenerative events in $\mathrm{AD}$ could be the impairment of growth factor signaling such as early insulin receptor desensitization (Hölscher, 2011).

\section{Link between diabetes mellitus and Alzheimer's disease}

One mechanism linking $\mathrm{DM}$ and $\mathrm{AD}$ is the alteration in brain insulin signaling. It has been shown that insulin signaling is involved in a variety of neuronal functions, and that it also plays a significant role in the pathophysiology of AD. Thus, the modification of neuronal insulin signaling by diabetic conditions may contribute to $\mathrm{AD}$ progression. Another possible mechanism is cerebrovascular alteration, a common pathological change observed in both diseases. Accumulating evidence has suggested the importance of $A \beta$ induced cerebrovascular dysfunction in $\mathrm{AD}$, and indicated that pathological interactions between the receptor for advanced glycation end products (RAGE) and $A \beta$ peptides may play a role in this dysfunction (Takeda et al., 2011).

Although the molecular mechanisms are not clear, it has been suggested that a complex interaction between T2DM and brain aging may occur, involving a decrease in brain insulin levels and/or action, impairment in brain glucose uptake and metabolism (despite peripheral hyperglycemia), mitochondrial dysfunction and oxidative stress. Increased AGEs formation and interaction with their receptors (RAGE) and impaired neurotransmission may be also involved. Decreased neurogenesis and neurodegeneration/death underlie the brain atrophy and cognitive dysfunction seen in both pathologies. Interestingly, GLP-1 mimetics have been shown to exert neuroprotective/neurogenesis effects against deleterious conditions such as $\mathrm{AD}$, besides their well-known insulinotropic and anorexigenic properties (Duarte et al., 2013) (Fig. 2).

\section{Biochemical Parameters}

\subsection{Acetylcholine (ACh)}

Altered levels of $\mathrm{ACh}$ and butyrylcholine $(\mathrm{BuCh})$ in $\mathrm{AD}$ and $\mathrm{T} 2 \mathrm{DM}$ implicate these two neurotransmitters in the pathogenesis of the two diseases. $\mathrm{AD}$ and $\mathrm{T} 2 \mathrm{DM}$ are characterized by elevated plasma Ach and BuCh. On the other hand, in $\mathrm{AD}$ the brain levels of $\mathrm{ACh}$ are decreased while those of $\mathrm{BuCh}$ increased, causing deregulation of the balance between $\mathrm{ACh}$ and $\mathrm{BuCh}$. 


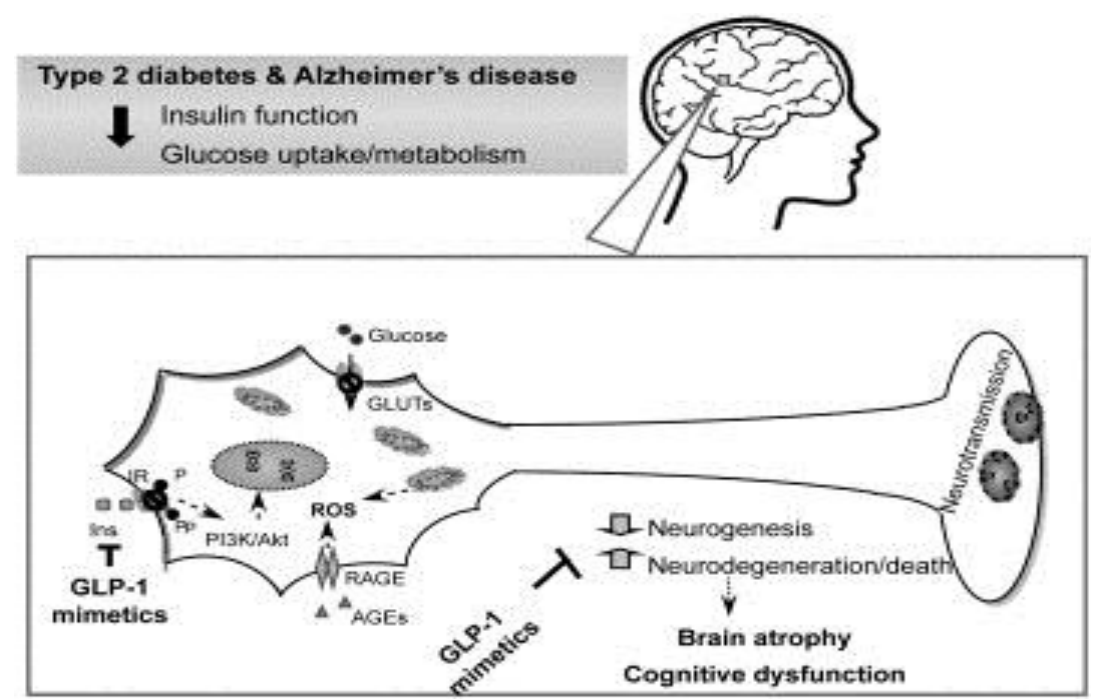

Figure. 2. Molecular mechanisms underlying long-term T2DM in CNS and AD

(Duarte et al., 2013).

The change in the $\mathrm{ACh} / \mathrm{BuCh}$ ratio causes cholinergic deficit in the brain, i.e. deficiency in the brain neurotransmitter ACh. General cholinesterase inhibitors that inhibit both AChE and BuChE as well as highly selective BuChE inhibitors may have potential therapeutic benefits in the treatment of $\mathrm{AD}$ and other dementias. A region near the C-terminus of human $\mathrm{AChE}$ is homologous to the $\mathrm{N}$-terminus of $\mathrm{AD} A \beta$ peptide. This further suggests that $\mathrm{AChE}$ may be involved in the formation of amyloid fibrils in senile plaques. The resulting $\mathrm{A} \beta-\mathrm{AChE}$ complexes were larger than controls injected with the same amount of $A \beta$ peptide alone. These $A \beta$ $\mathrm{AChE}$ complexes were more neurotoxic than the $\mathrm{A} \beta$ aggregates (Mushtaq et al., 2014).

Due to the absence of negative feedback control of acetylcholine, the decrease of $\mathrm{AChE}$ concentration leads to an increase of interleukin-1 (IL-1) and tumor necrotic factor (TNF- $\alpha)$. Therefore, the observation that the actions of AChase and BChase are increased in the plasma and red blood cells of T2DM and AD patients suggests that these proteins may be possible biochemical markers of these diseases (Dar et al., 2014).

Fig. 3 summarizes the role of acetylcholine as a molecular bond between $\mathrm{AD}$ and T2DM

\subsection{Homocysteine}

An increase in Hcy plasma levels is associated with several cognitive disorders, including cognitive decline associated with age, cerebrovascular disease, stroke and vascular dementia. Elevated plasma Hcy is an independent risk factor for $\mathrm{AD}$. Elevations in plasma Hcy temporally precede the development of dementia and there is a linear inverse relationship between plasma Hcy and cognitive performance in the elderly (Seshadri, 2006; Smith and Refsum, 2016).

Several studies have reported a positive association between plasma insulin and insulin resistance and plasma Hcy levels (Troen, 2005; Kronenberg et al., 2009). There is a molecular link between Hcy levels and insulin signaling. Insulin has been shown to inhibit the irreversible metabolism of Hcy to cysteine through transsulfuration when methionine is in demand. Glucose intolerance in animals given a diet rich in methionine suggests that serum Hcy levels may also modulate the sensitivity to insulin, leading to insulin resistance. In addition, hyperhomocysteinemia was correlated with a significant decrease in Akt phosphorylation (serine/threonine-specific protein kinase), which plays a major role in the insulin signaling pathway (Chiang et al., 2009).

\subsection{Lipid peroxidation}

One of the major targets of lipid peroxidation is the CNS. The brain is highly sensitive to oxidative stress; it consumes $20-30 \%$ of inspired oxygen, contains high levels of polyunsaturated fatty acids (PUFAs), is an ideal target for free radical attack 


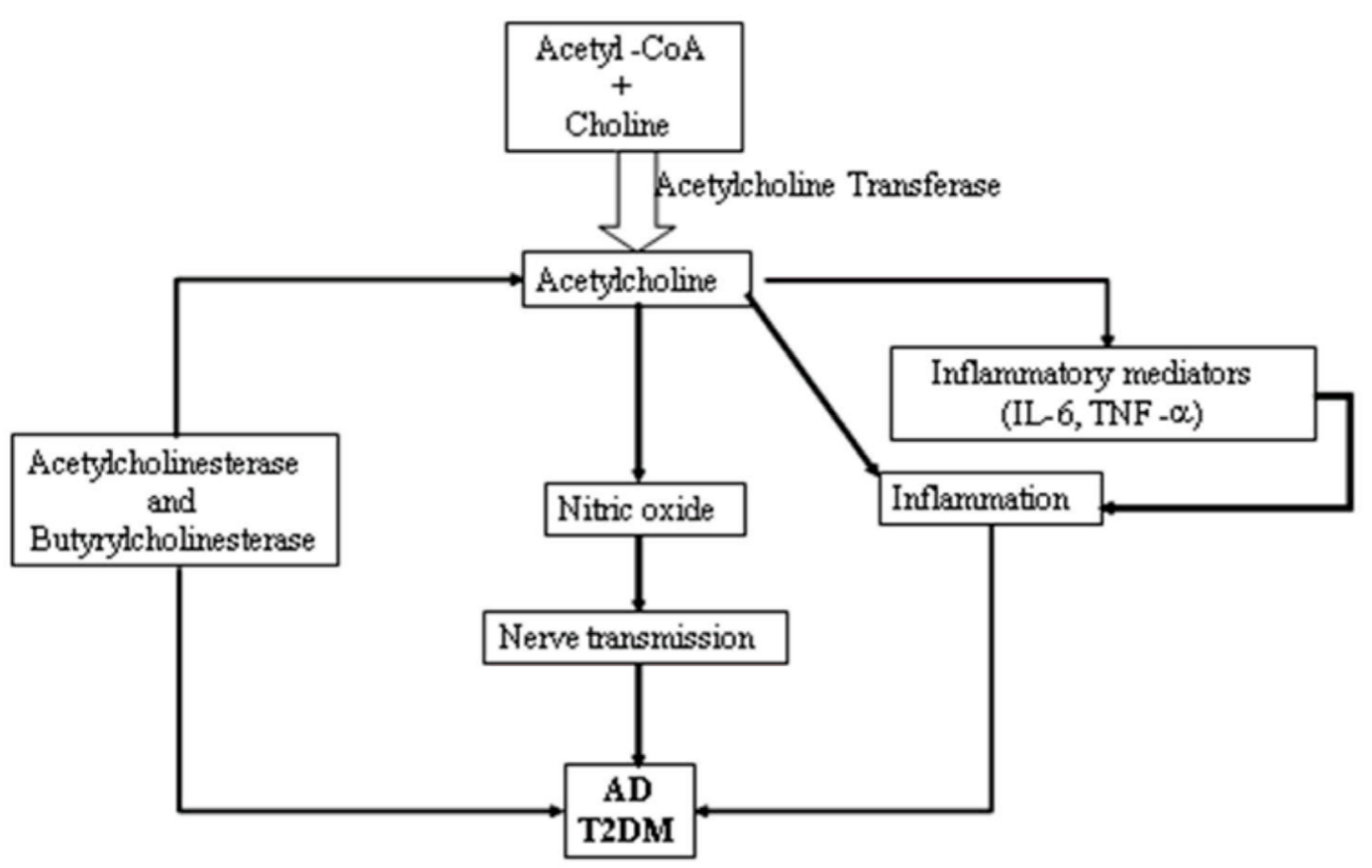

Figure 3. Acetylcholine as a molecular link between AD and T2DM (Dar et al., 2014).

and contains high levels of redox transition metals (Sultana et al., 2013). Oxidative stress, which is present in $\mathrm{AD}$, results in the formation of end products of free radical reactions with proteins and lipids. Therefore, specific products of lipid peroxidation in the blood of $\mathrm{AD}$ patients are present (Skoumalová et al., 2011).

Lipid hydroperoxides are unstable and in the presence of iron are non-enzymatically decomposed to a number of products, such as malondialdehyde (MDA) and 4-hydroxynonenal (4-HNE), ketones, epoxides and hydrocarbons. Aldehydes formed during lipid peroxidation of brain PUFA can diffuse from the primary sites and be used as markers of oxidative stress (Butterfield et al., 2006; Singh et al., 2010).

Another outcome of lipid peroxidation is the formation of isoprostanes. These are prostaglandinlike compounds formed from PUFA with at least three double bonds, including arachidonic and docosahexaenoic acid. Isoprostanes are produced in vivo by peroxidation of phospholipids nonenzymatically. F2-isoprostanes (F2-IsoPs) are formed from arachidonic acid via esterification with phospholipids followed by hydrolysis. In $\mathrm{AD}$, increased levels of F2-IsoPs were detected in CSF
(Montine, 2007; Leung et al., 2015).

F4-isoprostanes (F4-IsoPs) and structurally related to isoprostanes. They are products of radical peroxidation of docosahexaenoic acid, a highly prevalent PUFA in the brain. With six double bonds, docosahexaenoic acid is even more prone to free radical attack than arachidonic acid. Therefore, the detection of its peroxidative products is an important marker of brain oxidative damage and useful in neurodegenerative diseases. The level of F4-IsoPs was increased in CSF of AD patients compared with controls (Markesbery et al., 2005; Singh et al., 2010).

\subsection{ApoE \&4}

Diabetes is not only characterized by insulin/glucose abnormalities, but also by dyslipidemia. As key players in lipid metabolism, apolipoproteins are receiving growing attention for their involvement in T2DM (Jiang et al., 2017).

In $\mathrm{AD}$, apolipoprotein $\mathrm{E}$ (ApoE) is of interest, there is an increased risk for the development of earlier onset of $\mathrm{AD}$ with expression of apoE- $\varepsilon 4$ isoform. Along with proteoglycans and serum amyloid, ApoE is a nonfibrillar component of cerebral and systemic amyloid deposits (Hatters et al., 2006; Liu 
et al., 2016). Compared with other ApoE isoforms, apoE- $\varepsilon 4$ has an enhanced ability to deposit the neurotoxic $A \beta$ while simultaneously contributing to decreased clearance of plaque. While isomeric differences in the CNS have yet to be entirely elucidated, apoE- $\varepsilon 4$ exerts less protection against oxidative stress and contributes to cholinergic dysfunction in AD (Martins et al., 2009).

Furthermore, the lipid-binding capacity of ApoE, influenced by the cholesterol transporter ATP binding cassette (ABCA1), may have implications in $\mathrm{AD}$. Poor lipidation of ApoE, which results from transporter ABCA1 deficiency, causes a heavier amyloid burden, while overexpression of ABCA1 in the CNS (i.e. greater ApoE lipidation) results in significant reduction of $\mathrm{A} \beta$ plaque formation (Hirsch-Reinshagen et al., 2005; Corona et al., 2016).

There is also a negative correlation between the presence of the apoE- $\varepsilon 4$ isoform and expression of insulin degrading enzyme (IDE) in the brain; IDE is an endopeptidase that degrades cerebral $A \beta$. In nonapoE- $\varepsilon 4$ carriers, the IDE-A $\beta$ relationship is still relevant, as hyperinsulinaemia causes competitive inhibition of the binding (and hence, degradation) of A $\beta$ by IDE (Edland, 2004; Jha et al., 2015).

\subsection{Amyloid precursor protein}

Amyloid precursor protein (APP) is a type I transmembrane protein with a large extracellular amino-terminal domain, a transmembrane domain and an intracellular carboxy-terminal domain that resembles a cell surface receptor. It is expressed in several isoforms, with APP695 the main isoform expressed by neurons. APP has a high turnover, with a half-life ranging from 1 to 4 hours. APP undergoes extensive post-translational modification including glycosylation, phosphorylation, and tyrosine sulfation, as well as many types of proteolytic processing generating peptide fragments (Zhang et al., 2011; Wang et al., 2017).

There are four possible methods for APP processing; APP may remain functionally active at the cell surface; it may be internalised and recycled or degraded; it may be cleaved via the $\alpha$-secretase, releasing the sAPP $\alpha$ fragment, and a membrane bound C83 carboxy-terminal fragment that is further processed to the $\mathrm{P} 3$ fragment; or it may be cleaved via the $\beta$-secretase releasing $\operatorname{sAPP} \beta$, and a membrane bound C99 carboxy-terminal fragment that is further processed to the $A \beta$ fragment (Hunter and Brayne, 2012).
APP influences proliferation of neural progenitor cells and regulates transcriptional activity of various genes. App affects neurogenesis via two separate domains, soluble secreted APPs (sAPPs, mainly sAPP $\alpha$ ) and APP intracellular domain (AICD). The $\operatorname{sAPP} \alpha$ is neuroprotective and important to neurogenesis, whereas AICD negatively modulates neurogenesis. It has been reported that microRNA (miR-298, miR-106b and miR-328) can regulate APP expression, APP processing, $A \beta$ accumulation and subsequently neurotoxicity and neurogenesis related to APP, which is implicated in AD pathogenesis, especially sporadic AD. Secretase balances were proposed. Secretase balances can influence the downstream balance related to regulation of neurogenesis by AICD and sAPP $\alpha$ and balance of A $\beta$ and $\operatorname{sAPP} \alpha$ related to neuron viability. Disruption of these secretase balances are implicated in $\mathrm{AD}$ onset (Zhou et al., 2011).

Mutations in critical regions of APP, including the region that generates $A \beta$ cause familial susceptibility to AD. Several mutations outside the $A ß$ region in patients with familial Alzheimer increased production of $\mathrm{A} B$ (Zheng and Koo, 2006; Dawkins and Small, 2014).

\subsection{Amyloid beta (AB or Abeta)}

An amyloid is a deposits of proteins or plaques in the body. Extracellular amyloids are fibrous, insoluble protein aggregates with $\beta$ sheet structure. Amyloids are usually formed from misfolded proteins. The misfolded proteins can exist as monomers, dimers or oligomers. Amyloid plaques exist in different diseases, including neurodegenerative diseases, transmissible spongiform encephalopathies, T2DM, familial amyloidoses and other variants of systemic amyloidoses (Tycko and Wickner, 2013).

Beta amyloid $(A ß)$ is generated by the amyloidogenic pathway of APP processing. They are generated after $\beta$-secretase cleavage, followed by $\gamma$-secretase cleavage. A $\beta$ peptides vary in size, from 38 to 43 amino acids, with $A \beta 1-40$ being the predominant isoforms (90\%), and the more fibrilogenic A $\beta$ 1-42 accounting for $10 \%$ (Kametani, 2008; Deng et al., 2013).

Alzheimer's disease is caused in part by the overproduction and lack of clearance of $A \beta$ protein. Oligomer $A \beta$, the most toxic species of $A \beta$, causes direct injury to neurons, accompanied by increased neuroinflammation, astrocytosis and gliosis, and 
eventually neuronal loss (Rosenberg et al., 2016). Accumulation of cerebral $A \beta$ is essential for developing synaptic and cognitive deficits in AD. Endogenous $A \beta$ peptides are suggested to have a crucial role in activity-dependent regulation of synaptic vesicle release and which could be the primary pathological events that lead to compensatory synapse loss in AD (Abramov et al., 2009).

Oxidative stress mediated by reactive oxygen or nitrogen species (ROS/RNS) is implicated in several diseases including neurodegenerative diseases. In $\mathrm{AD}$, there is a large body of evidence that aggregation of $A \beta$ peptide is implicated in the generation of oxidative stress. Redox active metal ions play a key role in oxidative stress, either in the production of ROS/RNS by enzymes or loosely bound metals or in the protection against ROS, mostly as catalytic centers in enzymes. In $A D$, it is thought that metals (mostly $\mathrm{Cu}, \mathrm{Fe}$ and heme) can bind to $A \beta$ and that such systems are involved in the generation of oxidative stress (Chassaing et al., 2012).

Beta amyloid (A $\beta)$ modulates intracellular metabolic cascades, intracellular $\mathrm{Ca}^{2+}$ homeostasis and NMDA receptor expression in AD. High concentrations $(>2.5 \mu \mathrm{M} \mu \mathrm{mol})$ of $\mathrm{A} \beta$ cause neurotoxicity and cell death (Tanokashira et al., 2017). However, low levels ( $<200 \mathrm{pM})$ of $A ß$ to act as a trophic signal and modulate synaptic activities, with implications on memory and learning (Puzzo et al., 2008). The low levels of $A B$ could play an essential role in the regulation of plasticity of synapses and improvement of cognitive functions, while high $A ß$ concentrations together with age leads to dysregulation and loss of synaptic function (Gilson et al., 2015).

\subsection{Total tau and phospho tau}

Alzheimer disease and a family of neurodegenerative diseases called tauopathies are histopathologically characterized by a slow progressive neurodegeneration, which is associated primarily with the intracellular accumulation of tau (Iqbal et al., 2010; DeFelipe, 2016).

Tau protein was discovered in 20th century by studying factors necessary for microtubule formation. As illustrated in Fig. 4; tau protein stabilizes microtubules through four tubulin binding domains (blue boxes) in the longest isoform. Binding of tau protein to the microtubules is maintained in equilibrium by coordinated actions of kinases and phosphatases. The phosphorylation of tau (pink balls) regulates its activity to bind to microtubules and can affect axonal transport. Tau protein may inhibit the transport of end-over vesicles along microtubules by kinesin (Kolarova et al., 2012). In healthy neurons, tau binds to microtubules to regulate their stability; in $\mathrm{AD}$, tau is detached from microtubules and phosphorylated at multiple sites. Such abnormal tau behavior, which is triggered by $A \beta$, results in generation of pathological tau species that mediate neuron loss (Ando et al., 2016). Tau is a phosphoprotein with 79 potential Serine (Ser) and Threonine (Thr) phosphorylation sites on the longest tau isoform. Phosphorylation has been reported on approximately 30 of these sites in normal tau proteins. Phosphorylation of tau is regulated by a host of kinases, including PKN, a serine/threonine kinase.

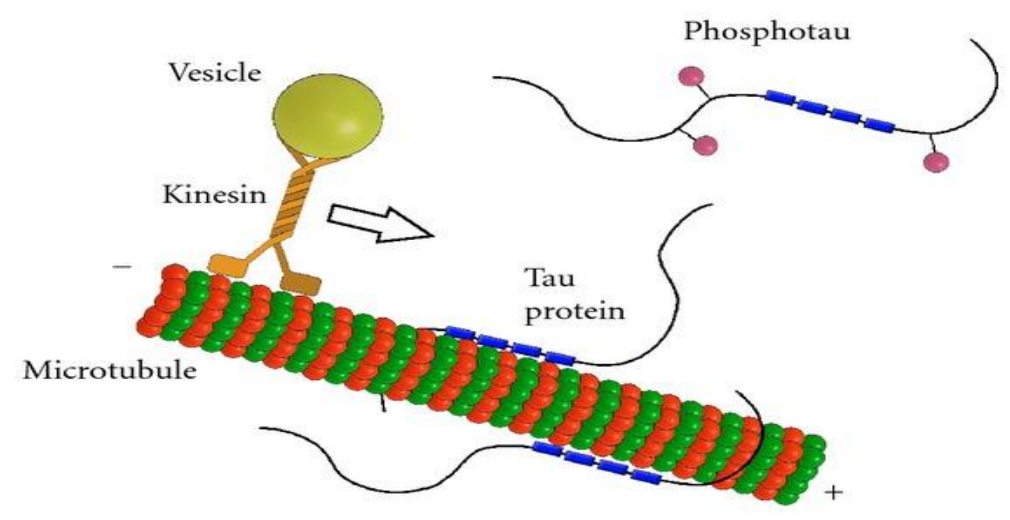

Figure 4. The structure and function of tau (Kolarova et al., 2012) 
When PKN is activated, it phosphorylates tau, resulting in disruption of microtubule organization. The microtubule-associated protein tau is a family of six isoforms that becomes abnormally hyperphosphorylated and accumulates in neurons undergoing neurodegeneration in the brains of patients with AD (Wang et al., 2013; Avila et al., 2014). As a major microtubule-associated protein, tau plays an important role in promoting microtubule assembly and stabilization. In $\mathrm{AD}$ and other tauopathies, the abnormally hyperphosphorylated tau proteins are aggregated

\section{References}

Abramov, E., Dolev, I., Fogel, H., Ciccotosto, G.D., Ruff, E., Slutsky, I., 2009. Amyloid-beta as a positive endogenous regulator of release probability at hippocampal synapses. Nat Neurosci. 12(12), 1567-1576.

American Diabetes Association, 2012. Diagnosis and classification of diabetes mellitus. Diabetes Care .35 (Suppl 1), S64-71.

Ando, K., Maruko-Otake, A., Ohtake, Y., Hayashishita, M., Sekiya, M., Iijima, K.M., 2016. Stabilization of Microtubule-Unbound Tau via Tau Phosphorylation at Ser262/356 by Par-1/MARK Contributes to Augmentation of AD-Related Phosphorylation and A $\beta 42$-Induced Tau Toxicity. PLoS Genet.12, e1005917.

Avila, J., Simón, D., Díaz-Hernández, M., Pintor, J., Hernández, F., 2014. Sources of extracellular tau and its signaling. J Alzheimers Dis. 40 Suppl 1, S7S15.

Burns, A., Iliffe, S., 2009. Alzheimer's disease. BMJ. 338, b158.

Butterfield, D.A., Reed, T., Perluigi, M., De Marco, C., Coccia, R., Cini, C., Sultana, R., 2006. Elevated protein-bound levels of the lipid peroxidation product, 4-hydroxy-2-nonenal, in brain from persons with mild cognitive impairment. Neurosci Lett. 3, 170-173.

Chassaing, S., Collin, F., Dorlet, P., Gout, J., Hureau, C., Faller, P., 2012. Copper and hememediated Abeta toxicity: redox chemistry, Abeta oxidations and anti-ROS compounds. Curr Top Med Chem .12(22), 2573-2595.

Chawla, A., Chawla, R., Jaggi, S., 2016. Micro- into paired helical filaments and accumulated in the neurons as neurofibrillary tangles (NFT). An imbalanced regulation in protein kinases and protein phosphatases is the direct cause of tau hyperphosphorylation. Among kinases and phosphatases, glycogen synthase kinase-3 $\beta$ (GSK$3 \beta$ ) and protein phosphatase $2 \mathrm{~A}(\mathrm{PP} 2 \mathrm{~A})$ are the most implicated. Accumulation of the hyperphosphorylated tau induces synaptic toxicity and cognitive impairments (Iqbal and GrundkeIqbal, 2008; Ma et al., 2017).

vasular and macrovascular complications in diabetes mellitus: Distinct or continuum? Indian J Endocrinol Metab. 20(4), 546-551.

Chiang, E.P., Wang, Y.C., Chen, W.W., Tang, F.Y., 2009. Effects of Insulin and Glucose on Cellular Metabolic Fluxes in Homocysteine Transsulfuration, Remethylation, SAdenosylmethionine Synthesis, and Global Deoxyribonucleic Acid Methylation. J Clin. Endocrinol Metab. 94, 1017-1025.

Corona, A.W., Kodoma, N., Casali, B.T., Landreth, G.E., 2016. ABCA1 is Necessary for BexaroteneMediated Clearance of Soluble Amyloid Beta from the Hippocampus of APP/PS1 Mice. J Neuroimmune Pharmacol. 11(1), 61-72.

DalForno, G., Palermo, M.T., Donohue, J.E., Karagiozis, H., Zonderman, A.B., Kawas, C.H., 2005. Depressive symptoms, sex, and risk for Alzheimer's disease. Ann Neurol. 57, 381-387.

Dar, T.A., Sheikh, I.A., Ganie, S.A., Ali, R., Singh, L.R., Gan, S.H., Kamal, M.A., Zargar, M.A., 2014. Molecular Linkages between Diabetes and Alzheimer's disease Current Scenario and Future Prospects. CNS Neurol Disord Drug Targets. 13(2), 290-298.

Dawkins, E., Small, D.H., 2014. Insights into the physiological function of the $\beta$-amyloid precursor protein: beyond Alzheimer's disease. J Neurochem. 129(5), 756-679.

De Nazareth, A.M., 2017.Type 2 diabetes mellitus in the pathophysiology of Alzheimer's disease. Dement Neuropsychol. 11 (2), 105-113.

DeFelipe, J., 2016. Phospho-Tau and Cognitive 
Decline in Alzheimer's Disease. Commentary: Tau in physiology and pathology. Front Neuroanat. 1044.

Deng, Y., Wang, Z., Wang, R., Zhang, X., Zhang, S., Wu, Y., Staufenbiel, M., Cai, F., Song, W., 2013. Amyloid- $\beta$ protein (A $\beta)$ Glu 11 is the major $\beta$ secretase site of $\beta$-site amyloid- $\beta$ precursor proteincleaving enzyme 1(BACE1), and shifting the cleavage site to $A \beta$ Asp1 contributes to Alzheimer pathogenesis. Eur J Neurosci. 37, 1962-1969.

Donix, M., Ercoli, L.M., Siddarth, P., Brown, J.A., Martin-Harris, L., Burggren, A.C., Miller, K.J., Small, G.W., Bookheimer, S.Y., 2012. Influence of Alzheimer disease family history and genetic risk on cognitive performance in healthy middle-aged and older people. Am J Geriatr Psychiatry. 20(7), 565-573.

Du, X., Wang, X., Geng, M., 2018. Alzheimer's disease hypothesis and related therapies. Transl Neurodegener. 7, 2.

Duarte, A.I., Candeias, E., Correia, S.C., Santos, R.X., Carvalho, C., Cardoso, S., Plácido, A., Santos, M.S., Oliveira, C.R., Moreira, P.I., 2013. Crosstalk between diabetes and brain: Glucagon-like peptide1 mimetics as a promising therapy against neurodegeneration. Biochim Biophys Acta. 1832(4), 527-541.

Edland, S.D., 2004. Insulin-degrading enzyme, apolipoprotein E, and Alzheimer's disease. J Mol Neurosci. 23, 213-217.

Ferreira-Vieira, T.H., Guimaraes, I.M., Silva, F.R., Ribeiro, F.M., 2016. Alzheimer's disease: Targeting the Cholinergic System. Curr Neuropharmacol. 14(1), 101-115.

GBD 2013 Mortality and Causes of Death Collaborators, 2015. Global, regional, and national age-sex specific all-cause and cause-specific mortality for 240 causes of death, 1990-2013: a systematic analysis for the Global Burden of Disease Study 2013. Lancet. 385(9963), 117-171.

Gilson, V., Mbebi-Liegeois, C., Sellal, F., deBarry, J., 2015. Effects of Low Amyloid- $\beta$ (A $\beta)$ Concentration on $A \beta 1-42$ Oligomers Binding and GluN2BMembrane Expression. J Alzheimers Dis. 47(2), 453-466.

Goldman, J.S., Hahn, S.E., Catania, J.W., LaRusseEckert, S., Butson, M.B., Rumbaugh, M., Strecker,
M.N., Roberts, J.S., Burke, W., Mayeux, R., Bird, T., 2011. Genetic counseling and testing for Alzheimer disease: joint practice guidelines of the American College of Medical Genetics and the National Society of Genetic Counselors. Genet Med. 13(6), 597-605.

Grossman, H., Bergmann, C., Parker, S., 2006. Dementia: a brief review. Mt Sinai J Med. 73, 985992.

Hanger, D.P., Anderton, B.H., Noble, W., 2009. Tau phosphorylation: the therapeutic challenge for neurodegenerative disease. Trends Mol Med. 15 (3), 112-119.

Hatters, D.M., Zhong, N., Rutenber, E., Weisgraber, K.H., 2006. Amino-terminal domain stability mediates apolipoprotein $\mathrm{E}$ aggregation into neurotoxic fibrils. J Mol Biol. 361, 932-944.

Head, E., Lott, I.T., Wilcock, D.M., Lemre, C.A., 2016. Aging in Down Syndrome and the Development of Alzheimer's .Disease Neuropathology. Curr Alzheimer Res. 13, 18-29.

Hirsch-Reinshagen, V., Maia, L.F., Burgess, B.L., Blain, J.F., Naus, K.E., McIsaac, S.A., Parkinson, P.F., Chan, J.Y., Tansley, G.H., Hayden, M.R., Poirier, J., Van, W., Wellington, C.L., 2005. The absence of ABCA1 decreases soluble ApoE levels but does not diminish amyloid deposition in two murine models of Alzheimer disease. J Biol Chem. 280, 43243-43256.

Hölscher, C., 2011. Diabetes as a risk factor for Alzheimer's disease: insulin signalling impairment in the brain as an alternative model of Alzheimer's disease. Biochem Soc Trans. 39(4), 891-897.

Honjo, K., Black, S.E., Verhoeff, N.P., 2012. Alzheimer's disease, cerebrovascular disease, and the $\beta$-amyloid cascade. Can J Neurol Sci. 39(6), 712-728

Hunter, S., Brayne, C., 2012. Relationships between the amyloid precursor protein and its various proteolytic fragments and neuronal systems. Alzheimers Res Ther. 4(2), 10.

Iqbal, K., Grundke-Iqbal, I., 2008. Alzheimer neurofibrillary degeneration: significance, etiopathogenesis, therapeutics and prevention: Alzheimer review series. J Cell Mol Med. 12(1), 38-55.

Iqbal, K., Liu, F., Gong, C.X., Grundke-Iqbal, I., 
2010. Tau in Alzheimer disease and related tauopathies. Curr Alzheimer Res. 7(8), 656-664.

Jha, N.K., Jha, S.K., Kumar, D., Kejriwal, N., Sharma, R., Ambasta, R.K., Kumar, P., 2015. Impact of Insulin Degrading Enzyme and Neprilysin in Alzheimer's Disease Biology: Characterization of Putative Cognates for Therapeutic Applications. J Alzheimers Dis. 48(4), 891-917.

Jiang, Y., Ma, L., Han, C., Liu, Q., Cong, X., Xu, Y., Zhao, T., Li, P., Cao, Y., 2017. Effects of Apolipoprotein E Isoforms in Diabetic Nephropathy of Chinese Type 2 Diabetic Patients. J Diabetes Res. 2017, 3560920.

Kametani, F., 2008. Epsilon-secretase: reduction of amyloid precursor protein epsilon-site cleavage in Alzheimer's disease. Curr Alzheimer Res. 5(2), 165171.

Knowler, W.C., Fowler, S.E., Hamman, R.F., Christophi, C.A., Hoffman, H.J., Brenneman, A.T., Brown-Friday, J.O., Goldberg, R., Venditti, E., Nathan, D.M., 2009. Diabetes Prevention Program Research Group. 10-year follow-up of diabetes incidence and weight loss in the Diabetes Prevention Program Outcomes Study. Lancet. 374, 1677-1686.

Kolarova, M., García-Sierra, F., Bartos, A., Ricny, J., Ripova, D., 2012. Structure and pathology of tau protein in Alzheimer disease. Int J Alzheimers Dis. 2012, 731526.

Kronenberg, G., Colla, M., Endres, M., Folic acid, neurodegenerative and neuropsychiatric disease. Curr Mol Med. 9(3), 315-323.

Leung, K.S., Galano, J.M., Durand, T., Lee, J.C., 2015. Current development in non-enzymatic lipid peroxidation products, isoprostanoids and isofuranoids, in novel biological samples. Free Radic Res. 49(7), 816-826.

Liu, C.C., Kanekiyo, T., Xu, H., Bu, G., 2013. Apolipoprotein E and Alzheimer disease: risk, mechanisms and therapy. Nat Rev Neurol. 9(2), 106-118.

Luchsinger, J.A., 2010. Diabetes, related conditions, and dementia. J Neurol Sci. 299(1-2), 35-38.

Ma, R.H., Zhang, Y., Hong, X.Y., Zhang, J.F., Wang, J.Z., Liu, G.P., 2017. Role of microtubuleassociated protein tau phosphorylation in Alzheimer's disease. J Huazhong Univ Sci
Technolog Med Sci. 37, 307-312.

Markesbery, W.R., Kryscio, R.J., Lovell, M.A., Morrow, J.D., 2005. Lipid peroxidation is an early event in the brain in amnestic mild cognitive impairment. Ann Neurol. 5, 730-735.

Martins, I.J., Berger, T., Sharman, M.J., Verdile, G., Fuller, S.J., Martins, R.N., 2009. Cholesterol metabolism and transport in the pathogenesis of Alzheimer's disease. J Neurochem. 111, 12751308.

McMurray, J.J., Holman, R.R., Haffner, S.M., Bethel, M.A., Holzhauer, B., Hua, T.A., Belenkov, Y., Boolell, M., Buse, J.B., Buckley, B.M., Chacra, A.R., Chiang, F.T., Charbonnel, B., Chow, C.C., Davies, M.J., Deedwania, P., Diem, P., Einhorn, D., Fonseca, V., Fulcher, G.R., Gaciong, Z., Gaztambide, S., Giles, T., Horton, E., Ilkova, H., Jenssen, T., Kahn, S.E., Krum, H., Laakso, M., Leiter, L.A., Levitt, N.S., Mareev, V., Martinez, F., Masson, C., Mazzone, T., Meaney, E., Nesto, R., Pan, C., Prager, R., Raptis, S.A., Rutten, G.E., Sandstroem, H., Schaper, F., Scheen, A., Schmitz, O., Sinay, I., Soska, V., Stender, S., Tamás, G., Tognoni, G., Tuomilehto, J., Villamil, A.S., Vozár, J., Califf, R.M., 2010. Effect of valsartan on the incidence of diabetes and cardiovascular events. N Engl J Med. 362, 1477-1490.

Michael, J., Fowler, M.D., 2008. Microvascular and Macrovascular Complications of Diabetes. Clinical Diabetes. 26 (2), 77-82.

Mitsuru, S., Naoyuki,S. 2017. Bidirectional interactions between diabetes and Alzheimer's disease. Neurochemistry International.108, 296302.

Mondragón-Rodríguez, S., Perry, G., Zhu, X., Moreira, P.I., Acevedo-Aquino, M.C., Williams, S., 2013. Phosphorylation of tau protein as the link between oxidative stress, mitochondrial dysfunction, and connectivity failure: implications for Alzheimer's disease. Oxid Med Cell Longev. 2013, 940603.

Montine, T.J., Quinn, J., Kaye, J., Morrow, J.D., 2007. Isoprostanes as biomarkers of late-onset Alzheimer's disease. J Mol Neurosci. 33, 114-119.

Mushtaq, G., Greig, N.H., Khan, J.A., Kamal, M.A., 2014. Status of acetylcholinesterase and butyrylcholinesterase in Alzheimer's disease and type 2diabetes mellitus. CNS Neurol Disord Drug Targets. 3(8), 1432-1439. 
Paisley, P., Serpell, M., 2017. Improving pain control in diabetic neuropathy. Practitioner. 261(1802), 23-26.

Prina, A.M., Mayston, R., Wu, Y.T., Prince, M., 2019. A review of the $10 / 66$ dementia research group. Soc Psychiatry Psychiatr Epidemiol. 54, 110

Puzzo, D., Privitera, L., Leznik, E., Fà, M., Staniszewski, A., Palmeri, A., Arancio, O., 2008. Picomolar amyloid-beta positively modulates synaptic plasticity and memory in hippocampus. $\mathrm{J}$ Neurosci. 28(53), 14537-1445.

Quattropani, M.C., Lenzo, V., Armieri, V., Filastro, A., 2018. The origin of depression in Alzheimer disease: a systematic review. Riv Psichiatr. 53(1), 18-30.

Rankin, C.A., Sun, Q., Gamblin, T.C., 2005. Pseudo-phosphorylation of tau at Ser202 and Thr205 affects tau filament formation. Brain Res Mol Brain Res. 138(1), 84-93.

Reitz, C., 2012. Alzheimer's disease and the amyloid cascade hypothesis: a critical review. Int J Alzheimers Dis. 2012, 369808.

Rodriguez, A., Muller, D.C., Engelhardt, M., Andres, R., 2005. Contribution of impaired glucose tolerance in subjects with the metabolic syndrome in the Baltimore Longitudinal Study of Aging. Metabolism. 54(4), 542-547.

Rosenberg, R.N., Lambracht-Washington, D., Yu, G., Xia, W., 2016. Genomics of Alzheimer Disease: A Review. JAMA Neurol. 73, 867-874.

Serrano-Pozo, A., Frosch, M.P., Masliah, E., Hyman, B.T., 2011. Neuropathological alterations in Alzheimer disease. Cold Spring Harb Perspect Med. 1(1), a006189.

Seshadri, S., 2006. Elevated plasma homocysteine levels: risk factor or risk marker for the development of dementia and Alzheimer's disease? J Alzheimers Dis. 9, 393-398.

Singh, M., Dang, T.N., Arseneault, M., Ramassamy, C., 2010. Role of by-products of lipid oxidation in Alzheimer's disease brain: a focus on acrolein. J Alzheimers Dis. 21(3), 741-756.

Skoumalová, A., Ivica, J., Santorová, P., Topinková, E., Wilhelm, J., 2011. The lipid peroxidation products as possible markers of
Alzheimer's disease in blood. Exp Gerontol. 46(1), 38-42.

Smith, A.D., Refsum, H., 2016. Homocysteine, B Vitamins, and Cognitive Impairment. Annu Rev Nutr. 36, 211-239.

Sultana, R., Perluigi, M., Butterfield, D.A., 2013. Lipid peroxidation triggers neurodegeneration: A redox proteomics view into the Alzheimer disease. Free Radic Biol Med. 62, 157-169.

Takeda, S., Sato, N., Rakugi, H., Morishita, R., 2011. Molecular mechanisms linking diabetes mellitus and Alzheimer disease: betaamyloid peptide, insulin signaling, and neuronal function. Mol BioSyst. 6, 1822-1827.

Tanokashira, D., Mamada, N., Yamamoto, F., Taniguchi, K., Tamaoka, A., Lakshmana, M.K., Araki, W., 2017. The neurotoxicity of amyloid $\beta$ protein oligomers is reversible in a primary neuron model. Mol Brain. 10, 4.

Troen, A.M., 2005. The central nervous system in animal models of hyperhomocysteinemia. Prog Neuropsychopharmacol Biol Psychiatry. 29, 11401151

Tycko, R., Wickner, R.B., Molecular structures of amyloid and prion fibrils: consensus versus controversy. Acc Chem Res. 46(7), 1487-1496.

Wang, J.Z., Xia, Y.Y., Grundke-Iqbal, I., Iqbal, K., 2013. Abnormal hyperphosphorylation of tau: sites, regulation, and molecular mechanism of neurofibrillary degeneration. $\mathrm{J}$ Alzheimers Dis. 33(Suppl 1), S123-139.

Wang, X., Zhou, X., Li, G., Zhang, Y., Wu, Y., Song, W, 2017. Modifications and Trafficking of APP in the Pathogenesis of Alzheimer's Disease. Front Mol Neurosci. 10, 294

Xue, H., Sun, Q., Liu, L., Zhou, L., Liang, R., He, R., Yu, H., 2017. Risk factors of transition from mild cognitive impairment to Alzheimer's disease and death: A cohort study. Compr Psychiatry. 78, 91-97.

Ye, J., 2013. Mechanisms of insulin resistance in obesity. Front Med. 7(1), 14-24.

Zhang, Y.W., Thompson, R., Zhang, H., Xu, H., 2011. APP processing in Alzheimer's disease. Mol Brain. 4, 3. 
Zheng, H., Koo, E.H., 2006. The amyloid precursor protein: beyond amyloid. Mol Neurodegener. 3, 1-5

Zhou, Z.D., Chan, C.H., Ma, Q.H., Xu, X.H., Xiao,.
Z.C., Tan, E.K., 2011. The roles of amyloid precursor protein (APP) in neurogenesis: Implications to pathogenesis and therapy of Alzheimer disease. Cell Adh Migr. 5(4), 280-292. 